\title{
Medium-term results following arthroscopic reduction in walking-age children with developmental hip dysplasia after failed closed reduction
}

\author{
Liang Zhao, Hua Yan, Changsheng Yang, Daozhang Cai and Yijun Wang*
}

\begin{abstract}
Background: Arthroscopic reduction has become increasingly popular as an alternative to open reduction for the treatment of developmental dysplasia of the hip (DDH). However, patient outcomes beyond one and a half years after surgery remain unclear. The purpose of this study is to report the medium-term outcomes of walking-age patients who received arthroscopic reduction after an unsuccessful closed reduction. This research was conducted as part of a retrospectively registered study.

Methods: We performed arthroscopic reduction in eight children with DDH after failed closed reduction between January 2010 and January 2012 and followed all cases for a minimum of 5 years. Arthroscopic reduction was performed using a two-portal approach without traction. Capsular release and resection of the transverse acetabular ligament were also performed if needed. Patient demographics, clinical variables, anatomical assessment measures, and post-operative complications were extracted from medical records.

Results: We treated five male and three female patients with an average age at operation of 15.6 months (range, 12 to 22 months). All obstacles to reduction were corrected arthroscopically. Concentric reduction of the hip joint was observed in post-operative $X$-rays in all cases. The average safe zone was increased from $17.5^{\circ}\left(8^{\circ}\right.$ to $\left.30^{\circ}\right)$ to $42.1^{\circ}\left(36^{\circ}\right.$ to $\left.50^{\circ}\right)$ after the operation. The average acetabular (AC) index was reduced from $40.3^{\circ}\left(33^{\circ}\right.$ to $\left.65^{\circ}\right)$ to 21. $9^{\circ}\left(19^{\circ}\right.$ to $\left.26^{\circ}\right)$ at the end of follow-up. No complications occurred and no patients developed necrosis of the femoral head, recurrent dislocation, or residual hip dysplasia.

Conclusions: Arthroscopic reduction is a suitable surgical procedure for the treatment of DDH among walkingage children with failed closed reduction and severe dislocation. This method is quick and safe, and it can be performed without post-operative complications over the medium term.
\end{abstract}

Keywords: Developmental dysplasia of hip, Unsuccessful closed reduction, Arthroscopic reduction

\section{Introduction}

Developmental dysplasia of the hip (DDH) is a relatively common hip deformity among infants. Early detection and treatment of DDH are critical to avoid the risk of disability [1]. The application of a Pavlik harness or a spica cast during the first week of life can be effective in most cases. Among infants and young children with

\footnotetext{
*Correspondence: wangyijun678@163.com

Department of Orthopedics, Academy of Orthopedics, Guangdong Province, The Third Affiliated Hospital of Southern Medical University, 183 Zhongshan Avenue West, Guangzhou 510630, China
}

$\mathrm{DDH}$, the success rate of early Pavlik harness treatment can be as high as $90 \%$ [2]. Unfortunately, many patients, especially those in developing countries, miss this early treatment window. Closed reduction at a later stage is associated with a higher failure rate and can lead to hip instability.

Open reduction, sometimes combined with acetabuloplasty and femoral osteotomy, remains the standard treatment after failed closed reduction [3-6]. However, serious complications $[7,8]$, including avascular necrosis of the femoral head, may occur following open reduction 
and negatively affect patient outcomes. Previous studies [9-11] have reported that the rate of necrosis can be as high as $69 \%$ if a medial approach is used and up to $30 \%$ if an anterior approach is used.

In the search for a less invasive alternative to open reduction, arthroscopic reduction has been performed in several studies [12-15] to treat children with DDH. For example, McCarthy and MacEwen [13] reported the outcomes of three patients with hip dysplasia who received arthroscopic reduction 9 months after the procedure. One patient developed residual dysplasia that required surgery. Eberhardt et al. [14] performed arthroscopic reduction on five very young infants and reported outcomes at a mean follow-up of 13.2 months. A later study by Eberhardt et al. [15] reported the experiences of nine walking-age children who received arthroscopic reduction and acetabuloplasty to treat dislocated hips with a mean follow-up of 15.4 months. However, patient outcomes after a longer time period remain unclear. To fill this research gap, our study assessed the medium-term outcomes of walking-age patients who underwent arthroscopic reduction after an unsuccessful closed reduction.

\section{Materials and methods}

\section{Study participants}

This was a prospective single-centre observational study. The study participants included eight children with DDH after failed closed reduction scheduled to undergo arthroscopic reduction between January 2010 and January 2012 at the Third Affiliated Hospital of Southern Medical University. Surgery indications included patients who underwent a failed closed reduction aged 12 to 24 months, with a magnetic resonance imaging (MRI) scan indicating the presence of intra-acetabular soft tissue or an inverted labrum. The study excluded children over 24 months of age and cases with hip infection (synovial fluid puncture), a comorbid condition (e.g., disease of immune system), or a history of hip surgery (such as acetabuloplasty).

\section{Medical procedure}

All patients were recruited to participate in the study and received an arthroscopic reduction for the treatment of dislocated hips. All procedures were performed by the same surgeon who has extensive experience in adult hip arthroscopic surgery. The procedure was performed under general anesthesia and in a supine position. Arthrography was conducted before the operation to assess the position of the femoral head in relation to other anatomical structures. Two portals without traction were used in all cases. A small pad was placed under the affected hemipelvis. Anatomical landmarks including the femoral artery, the femoral head, the anterior superior iliac spine, and the pubic symphysis were marked prior to incision. With the affected hip in a $90^{\circ}$ flexed and $40^{\circ}-60^{\circ}$ abducted position, three Kirschner wires were positioned in parallel spaced $0.5 \mathrm{~cm}$ apart and directed inward and downward to the pubic symphysis. The wires were placed above, at the same level of, and below the femoral head. Fluoroscopy was used to guide the initial portal placement. To reduce the arthroscopic puncture using a trocar and reduce the $\mathrm{X}$-ray radiation effects in children, three Kirschner wires were used because we only performed intraoperative fluoroscopy once, when we established an anterolateral portal. One of the three Kirschner wires can be used to position and direct the anterolateral portal's puncture. We marked direction; the trocar used for the arthroscopic puncture is able to accurately enter the hip.

After an anterolateral portal was marked, a spinal needle was inserted into the hip joint following the previously marked direction. After passing through the tough joint capsule, $20 \mathrm{ml}$ saline was injected. If the needle was successfully placed in the hip joint, the saline fluid was ejected from the needle after removing the syringe. Fluoroscopy was conducted to determine the depth of the spinal needle in the joint cavity. A mark was made on the arthroscope cannula to prevent articular cartilage damage caused by an excessively deep puncture in the joint cavity. A vertical incision of $1 \mathrm{~cm}$ was made in the skin with hemostatic forceps, which were used for subcutaneous blunt dissection. An arthroscope puncture trocar was introduced into the hip joint to the depth as marked, and a characteristic "pop" could be felt when penetrating the joint cavity. The arthroscopic sheath was inserted into the joint capsule along the sheath core. In addition, an anterior portal was created where the perpendicular line of the anterior superior iliac spine and the horizontal line of the pubic symphysis met.

Arthroscopy was performed using a $4.0-\mathrm{mm}, 30^{\circ}$ arthroscope. After introduction into the joint cavity, the arthroscope was turned laterally and then in the medial direction to examine the acetabular rim, ligamentum teres, and femoral head. An exploration was conducted to identify obstacles to reduction, including a hypertrophic ligamentum teres (Fig. 1a), fibrofatty or pulvinar tissues (Fig. 1b), and a hypertrophic acetabular labrum (Fig. 1c). The acetabular pulvinar tissue was removed using a shaver (Fig. 1d), and the hypertrophic acetabular labrum and the ligamentum teres was resected with an electrocautery probe (Fig. 1e). After these steps, the horseshoe-shaped articular surface and the acetabular fossa became visible. If a capsular constriction was present, a capsular release was performed with an electrocautery probe. Resection of the transverse acetabular ligament was performed if needed. The surgery lasted $50 \pm 10 \mathrm{~min}$ in all cases. Proper positioning of the femoral head and the acetabulum were confirmed by X-ray 

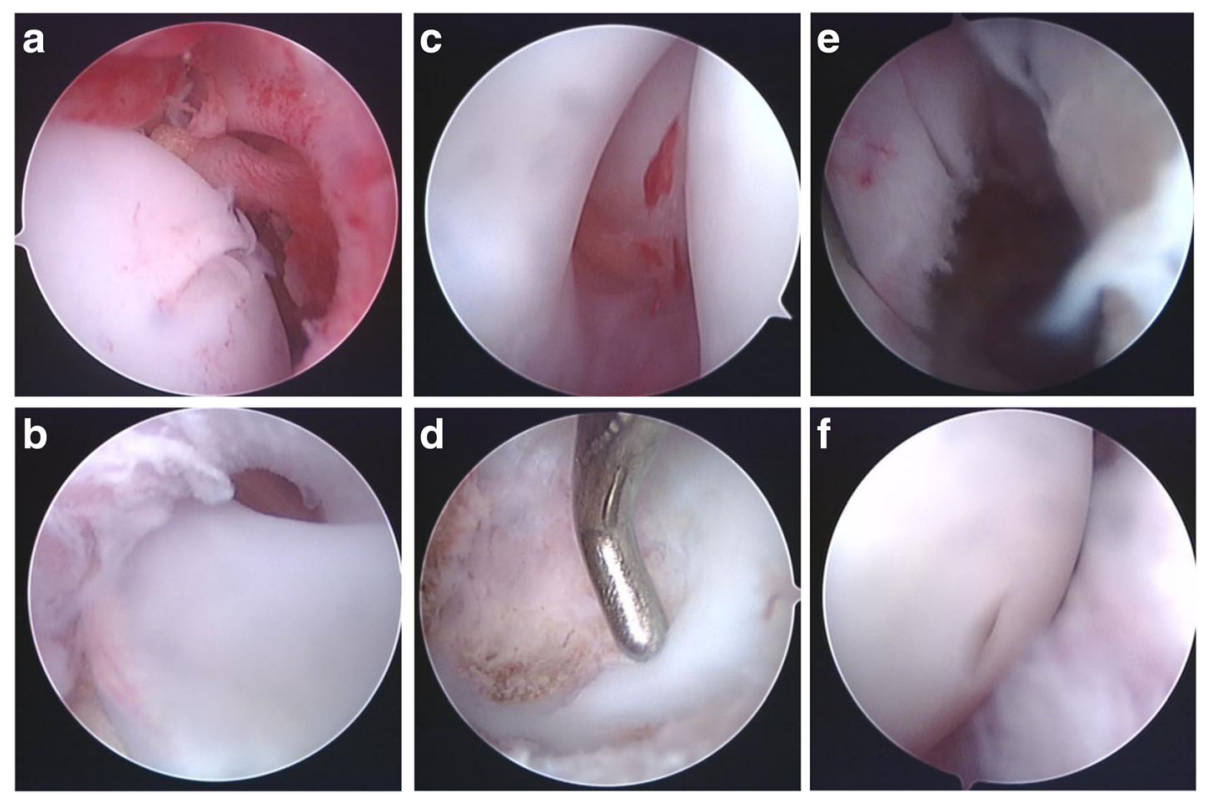

Fig. 1 Arthroscopic images of the hip joint. a A hypertrophic ligamentum teres. b Arthroscopic images of the acetabular fossa and pulvinar tissues. c Arthroscopic images of a hypertrophic acetabular labrum. d Resection of the acetabular fossa and pulvinar tissue using a shaver. e Resection of a hypertrophic acetabular labrum with an electrocautery probe. f Proper positioning of the femoral head and the acetabulum following arthroscopic reduction

following the arthroscopic reduction procedure (Fig. 1f). A spica cast was applied to retain the hip in a moderately flexed and abducted position for 12 weeks followed by the application of a Pavlik harness to maintain the reduction fixation for 3 to 6 months. During the first year of follow-up, doctor appointments and X-ray examinations were arranged every month. During the second year and thereafter, doctor appointments and X-ray examinations were conducted once per year.

The patient's gender, age at operation, affected side, previous treatments, and pre-operative Tönnis grade of dislocation were obtained. Anatomical measurements including the safe zone and the acetabular (AC) index were collected both before and after the operation. The $\mathrm{AC}$ index refers to the angle formed by Hilgenreiner's line and a line that extends along the acetabular roofs. A normal AC index is less than $30^{\circ}$. A safe zone was used to assess the stability of the hip joint after arthroscopic reduction, which is defined as the range between the maximum hip abduction angle and the maximum hip adduction angle without dislocation. Safe zone determination was carried out upon hip flexion at $90^{\circ}$ after hip joint reduction, followed by recording the full hip abduction angle and the thigh adduction angle to hip joint extrusion. A larger safe zone indicates better stability of the hip joint and vice versa. The ideal safe zone ranges from $30^{\circ}$ to $65^{\circ}[16]$. X-rays were obtained at different time points during the follow-up in all cases to monitor the reduction of the hip joint. The post-operative complications [17] included in the analysis were residual hip dysplasia, subluxation or repeated dislocation of the hip, and avascular necrosis of the femoral head.

\section{Statistical analysis}

Data were collected in a Microsoft Excel workbook. Descriptive statistics such as the mean, standard deviation, count, and percent are reported. Paired two-sample Student's $t$ tests were performed using SPSS 22.0 software (IBM, Armonk, NY, USA) to test the statistical significance of the changes in anatomical assessments before and after the operation.

\section{Results}

The study included five male and three female patients, with an average age at operation of 15.6 months (12 to 22 months). All patients were affected unilaterally with five affected hips on the right side and three on the left side. Before arthroscopic reduction, seven patients were treated unsuccessfully with open adductor tenotomy and a spica cast and one failed closed reduction through the application of a Pavlik harness, and all cases were performed serial radiographic studies to monitor whether the reduction is concentric or eccentric for 3 months at least. According to the Tönnis grade of dislocation, two grade III hips and six grade IV hips were included. The main obstacles to reduction included pulvinar tissue, a hypertrophic ligamentum teres, a hypertrophic transverse acetabular ligament, and capsular constriction, 
which were observed in all eight cases. An inverted labrum, which represents changes to the labrum cartilage complex but is not an obstacle to reduction, was observed in two hips. A pressure lesion in the cartilaginous acetabular roof was observed in all hips, and a neolimbus formation was observed in two hips. Patients' demographics and pre-operative characteristics are shown in Table 1.

Arthroscopic reduction, including the resection of the pulvinar tissue and ligamentum teres, transverse ligament incision, and capsule release, was performed unilaterally in all patients. All reduction obstacles could be arthroscopically eliminated. Concentric reduction of the hip joint was observed on post-operative X-rays in all cases. The average pre-operative safe zone was $17.5^{\circ}\left(8^{\circ}\right.$ to $\left.30^{\circ}\right)$, while the post-operative safe zone was $42.1^{\circ}\left(36^{\circ}\right.$ to $\left.50^{\circ}\right)$. Therefore, the safe zone increased by $24.6^{\circ}$ on average (95\% CI, $-30.7,-18.5, p<0.001)$ after the surgery. The patients were followed-up for a period of 60 months. The average pre-operative $\mathrm{AC}$ index was $40.3^{\circ}\left(33^{\circ}\right.$ to $\left.65^{\circ}\right)$. The average $\mathrm{AC}$ index at the final follow-up was $21.9^{\circ}\left(19^{\circ}\right.$ to $\left.26^{\circ}\right)$, which represents an average decrease of $18.4^{\circ}$ (95\% CI, 10.4, 26.4, $p<0.001$ ). No complications, such as wound hematoma, infection, and neurological or vascular injuries, occurred after surgery. During the follow-up period, none of the patients developed necrosis of the femoral head, and periodic Xray examinations showed continuous growth of the ossific nucleus of the femoral head. None of the patients developed a recurrent dislocation or residual hip dysplasia. X-ray images of one case before the operation (Fig. 2a) and during follow-up (Fig. 2b-h) are shown. The surgery results and complications during follow-up for individual patients are presented in Table 2.

\section{Discussion}

The treatment for dislocated hips depends on the age of the patient, the degree of dislocation, the anatomical configuration of the proximal femur, and the existing acetabular dysplasia. If the dislocated hip cannot be treated through closed reduction during the first year of life, more extensive treatment is necessary [18]. Although the open reduction remains the standard treatment after failed closed reduction, arthroscopic reduction has been performed in several studies. Despite the increasing number of reports of arthroscopically assisted reduction [13, 19], medium-term outcomes have yet to be reported. Our research findings suggest the medium-term effectiveness of arthroscopic reduction for walking-age children with DDH who failed closed reduction. Further follow-up is warranted to assess the long-term results, as the patients had not reached skeletal maturity.

Seven of eight of our patients previously underwent open adductor tenotomy and spica cast treatment, which failed to restore the femoral head-acetabulum concentricity. Evidence [20] indicates that the failure rate of this treatment can be up to $50 \%$. The high failure rate of closed reduction can be largely attributed to excessive intra-acetabular contents such as hypertrophic fibrofatty (pulvinar) tissue, a thickened ligamentum teres, and an inverted labrum. A small amount of the content may gradually disappear after closed reduction and lead to the formation of femoral head-acetabulum concentricity, allowing the hip joint to return to its normal morphology. In contrast, a large amount of intra-acetabular content will obstruct the repositioning of the femoral head into the acetabulum, which is a major cause of closed reduction failure [21]. One case in our sample failed Pavlik harness treatment. If radiographic assessments show that the hip is not responding to treatment within 3 weeks of application of the harness, the treatment should be discontinued [18].

Various surgical approaches [22-24] have been developed for hip arthroscopy. All arthroscopic reduction procedures in this study were conducted using a twoportal approach with an anterolateral portal and an anterior portal under Kirschner wire-assisted positioning of the hip joint. A similar two-portal approach has been adopted by previous studies $[14,15]$ to examine the anatomical structure within the hip joint and to perform arthroscopic reduction. To ensure proper positioning and portal placement and to avoid unnecessary soft tissue damage, we used a mobile $\mathrm{C}$-arm machine and multiple Kirschner wires to establish the hip surgical approaches in all cases. We were able to examine all key anatomical structures and remove all obstacles to reduction through the established portals.

The main obstacles to reduction observed in this study included pulvinar tissue, a hypertrophic ligamentum teres, hypertrophic transverse acetabular ligaments, and capsule constriction. These findings are consistent with previous reports $[14,15]$. We also observed pressure lesions in the cartilaginous acetabular roof in all cases and an inverted labrum and neolimbus in two cases. These rates are comparable to previously reported rates by Eberhardt et al. [15] who studied older pediatric patients with less severe dislocations.

Bulut et al. [19] reported a combination of arthroscopic reduction and open psoas tenotomy. Unlike Bulut et al., who performed an arthroscopically assisted procedure, we performed a purely arthroscopic reduction. We used arthrography to determine whether the reduction was concentric. All patients reported good outcomes. If the postarthroscopic reduction was not concentric, psoas tenotomy was performed. All obstacles to reduction were examined and eliminated arthroscopically. In all cases in this study, the hips could be repositioned and stably retained in a Pavlik harness and a spica cast without the use of psoas tenotomy. 


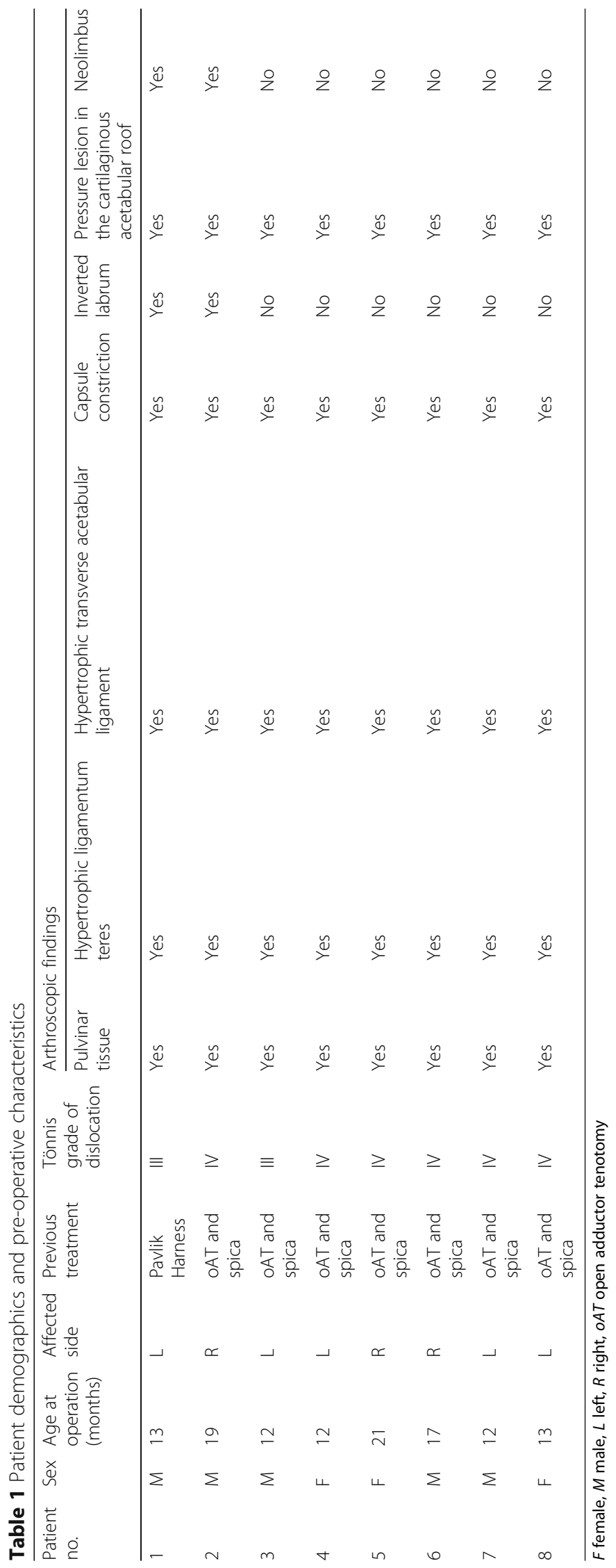



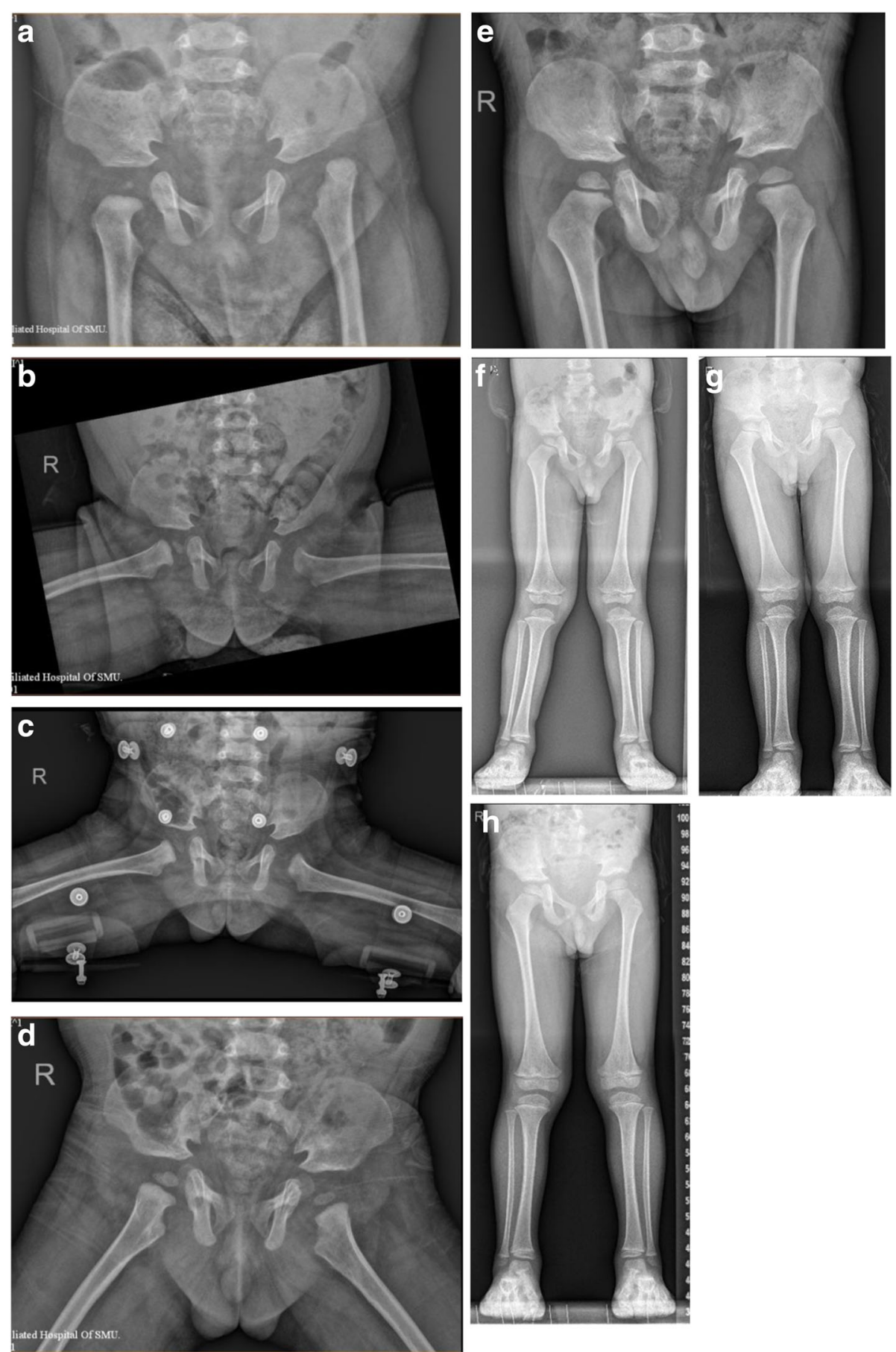

Fig. 2 X-ray images of one patient before the operation and during follow-up. a Pre-operational imaging. b Arthroscopic reduction (concentric reduction) at 1 day after the operation, $\mathbf{c} 3$ months of follow-up, $\mathbf{d} 12$ months of follow-up, e 2 years of follow-up, $\mathbf{f} 3$ years of follow-up, $\mathbf{g} 4$ years of follow-up, and $\mathbf{h}$ 5 years of follow-up

Although the medium-term results are encouraging and demonstrate the feasibility of arthroscopic reduction in treating walking-age children with DDH, some limitations of this study should be noted. First, our sample size was relatively small. Second, our study did not have a control group to compare the complication rates associated with other treatment options. Third, this case series reported a single surgeon's experience, and all cases included in this study were treated at one community hospital. Finally, the age of the included patients ranged from 1 to 2 years. Despite the limitations inherent to this case series, we present the medium-term 
Table 2 Surgery results and complications during follow-up

\begin{tabular}{|c|c|c|c|c|c|c|c|c|c|}
\hline \multirow{2}{*}{$\begin{array}{l}\text { Patient } \\
\text { no. }\end{array}$} & \multirow{2}{*}{$\begin{array}{l}\text { Concentric } \\
\text { reduction on } \\
\text { post-op X-ray }\end{array}$} & \multirow{2}{*}{$\begin{array}{l}\text { Pre-op safe } \\
\text { zone }\left({ }^{\circ}\right)\end{array}$} & \multirow{2}{*}{$\begin{array}{l}\text { Post-op safe } \\
\text { zone }\left(\left(^{\circ}\right)\right.\end{array}$} & \multirow{2}{*}{$\begin{array}{l}\text { Follow-up period } \\
\text { (months) }\end{array}$} & \multirow{2}{*}{$\begin{array}{l}\text { Pre-op } \\
\text { AC Index }\left(^{\circ}\right)\end{array}$} & \multirow{2}{*}{$\begin{array}{l}\text { AC Index at most recent } \\
\text { follow-up }\left(^{\circ}\right)\end{array}$} & \multicolumn{3}{|c|}{ Post-op complications } \\
\hline & & & & & & & $\begin{array}{l}\text { Necrosis of } \\
\text { the femoral } \\
\text { head }\end{array}$ & $\begin{array}{l}\text { Repeated } \\
\text { dislocation } \\
\text { of hip }\end{array}$ & $\begin{array}{l}\text { Residual } \\
\text { hip } \\
\text { dysplasia }\end{array}$ \\
\hline 1 & Yes & 18 & 43 & 48 & 35 & 19 & No & No & No \\
\hline 2 & Yes & 20 & 40 & 48 & 37 & 21 & No & No & No \\
\hline 3 & Yes & 17 & 45 & 48 & 33 & 24 & No & No & No \\
\hline 4 & Yes & 18 & 38 & 48 & 38 & 26 & No & No & No \\
\hline 5 & Yes & 19 & 40 & 48 & 38 & 23 & No & No & No \\
\hline 6 & Yes & 8 & 50 & 48 & 65 & 23 & No & No & No \\
\hline 7 & Yes & 10 & 45 & 42 & 36 & 20 & No & No & No \\
\hline 8 & Yes & 30 & 36 & 42 & 40 & 19 & No & No & No \\
\hline
\end{tabular}

AC Index acetabular index, Pre-op pre-operative, Post-op post-operative

results of arthroscopic reduction among walking-age children with severe hip dislocation. The results of this study are promising because no arthroscopic-associated complications occurred within an average follow-up period of 5 years. This study, together with other published studies [13-15], demonstrates that arthroscopic reduction is suitable for treating DDH among walkingage children, even those with severe dislocation. Further research that directly compares the results and complications of open reduction to arthroscopic reduction with longer follow-up periods and without psoas tenotomy will be necessary to confirm the efficacy of arthroscopic reduction.

\section{Conclusions}

Arthroscopic reduction is a suitable surgical procedure for the treatment of DDH among walking-age children with failed closed reduction and severe dislocation. It is quicker, safer, and can be achieved without post-operative complications over the medium term.

\section{Abbreviations}

AC index: Acetabular index; DDH: Developmental dysplasia of the hip; MRI: Magnetic resonance imaging; oAT: Open adductor tenotomy; Postop: Post-operative; Pre-op: Pre-operative

\section{Acknowledgements}

We are deeply grateful to all of the research staff, and this study would not be possible without their active participation and generous assistance.

\section{Funding}

No funding resources.

\section{Availability of data and materials}

Data sharing is not applicable to this article, as no data sets were generated or analyzed during the current study.

\section{Authors' contributions}

All the authors participated in the planning and design of the study and in the interpretation of the results. YW performed the operations. CY analyzed the data. LZ was responsible for writing the manuscript, and all authors actively participated in the preparation and revision of the article. All authors read and approved the final manuscript.
Ethics approval and consent to participate

The medical ethical committee of the Third Affiliated Hospital of Southern Medical University granted research ethics approval. The reference number is not applicable. We obtained informed consent from each patient before any testing was performed.

\section{Consent for publication}

Not applicable.

\section{Competing interests}

The authors declare that they have no competing interests.

\section{Publisher's Note}

Springer Nature remains neutral with regard to jurisdictional claims in published maps and institutional affiliations.

Received: 5 June 2017 Accepted: 13 September 2017

Published online: 21 September 2017

\section{References}

1. Eastwood DM. Neonatal hip screening. Lancet. 2003;361(9357):595-7.

2. Cashman JP, Round J, Taylor G, Clarke NM. The natural history of developmental dysplasia of the hip after early supervised treatment in the Pavlik harness. A prospective, longitudinal follow-up. J Bone Joint Surg Br. 2002;84(3):418-25. Epub 2002/05/11. PubMed PMID: 12002504

3. Arslan H, Kapukaya A, Ibrahim Bekler H, Necmioglu S. Is varus osteotomy necessary in one-stage treatment of developmental dislocation of the hip in older children? J Child Orthop. 2007;1(5):291-7. Epub 2007/11/01. doi: 10, 1007/s11832-007-0047-z. PubMed PMID: 19308523; PubMed Central PMCID: PMC2656742

4. Wenger DR, Mubarak SJ, Henderson PC, Miyanji F. Ligamentum teres maintenance and transfer as a stabilizer in open reduction for pediatric hip dislocation: surgical technique and early clinical results. J Child Orthop. 2008;2(3):177-85. Epub 2009/03/25. doi: 10.1007/s11832-008-0103-3. PubMed PMID: 19308575; PubMed Central PMCID: PMC2656808

5. Mootha AK, Saini R, Dhillon M, Aggarwal S, Wardak E, Kumar V. Do we need femoral derotation osteotomy in DDH of early walking age group? A clinico-radiological correlation study. Arch Orthop Trauma Surg. 2010;130(7): 853-8. Epub 2009/12/17. doi: 10.1007/s00402-009-1020-8 [doi]. PubMed PMID: 20012070

6. Gunel U, Daglar B, Tasbas BA, Delialioglu O, Bayrakci K. Results of Tonnistype acetabuloplasty in patients with developmental hip dysplasia. J Orthop Sci. 2012;17(1):705-9. Epub 2012/09/01. doi: 10.1007/s00776-012-0287-8. S0949-2658(15)30581-9 [pii]. PubMed PMID: 22936208

7. Roposch A, Ridout D, Protopapa E, Nicolaou N, Gelfer Y. Osteonecrosis complicating developmental dysplasia of the hip compromises subsequent acetabular remodeling. Clin Orthop Relat Res. 2013;471(7):2318-26. Epub 2013/01/29. doi: 10.1007/s11999-013-2804-2. PubMed PMID: 23354465; PubMed Central PMCID: PMC3676631 
8. Wu KW, Wang TM, Huang SC, Kuo KN, Chen CW. Analysis of osteonecrosis following Pemberton acetabuloplasty in developmental dysplasia of the hip long-term results. J Bone Joint Surg Am. 2010;92(11):2083-94. Epub 2010/ 09/03. doi: 10.2106/JBJS.I.01320. PubMed PMID: 20810858

9. Okano K, Yamada K, Takahashi K, Enomoto H, Osaki M, Shindo H. Long-term outcome of Ludloff's medial approach for open reduction of developmental dislocation of the hip in relation to the age at operation. Int Orthop. 2009; 33(5):1391-6. Epub 2009/05/19. doi: 10.1007/s00264-009-0800-7. PubMed PMID: 19449005; PubMed Central PMCID: PMC2899135

10. Pospischill R, Weninger J, Ganger R, Altenhuber J, Grill F. Does open reduction of the developmental dislocated hip increase the risk of osteonecrosis? Clin Orthop Relat Res. 2012;470(1):250-60. Epub 2011/06/07. doi: 10.1007/s11999-011-1929-4. PubMed PMID: 21643924; PubMed Central PMCID: PMC3237975

11. Morcuende JA, Mever MD, Dolan LA, Weinstein SL. Long-term outcome after open reduction through an anteromedial approach for congenital dislocation of the hip. J Bone Joint Surg Am. 1997;79(6):810-7. Epub 1997/ 06/01. PubMed PMID: 9199376

12. Ozturk H, Bulut O, Tezeren G, Oztemur Z. Hip arthroscopy for developmental dislocation. Orthopedics. 2007;30(8):600. Epub 2007/08/31. PubMed PMID: 17727011

13. McCarthy JJ, MacEwen GD. Hip arthroscopy for the treatment of children with hip dysplasia: a preliminary report. Orthopedics. 2007;30(4):262-4. Epub 2007/04/12. PubMed PMID: 17424686

14. Eberhardt O, Fernandez FF, Wirth T. Arthroscopic reduction of the dislocated hip in infants. J Bone Joint Surg Br. 2012;94(6):842-7. Epub 2012/ 05/26. doi: 10.1302/0301-620X.94B6.28161. PubMed PMID: 22628603

15. Eberhardt O, Wirth T, Fernandez FF. Arthroscopic reduction and acetabuloplasty for the treatment of dislocated hips in children of walking age: a preliminary report. Arch Orthop Trauma Surg. 2014;134(11):1587-94. Epub 2014/08/01. doi: 10.1007/s00402-014-2063-z. PubMed PMID: 25077783

16. Lewinnek GE, Lewis JL, Tarr R, Compere CL, Zimmerman JR. Dislocations after total hip-replacement arthroplasties. J Bone Joint Surg Am. 1978;60(2): 217-20. Epub 1978/03/01. PubMed PMID: 641088

17. Salter RB, Kostuik J, Dallas S. Avascular necrosis of the femoral head as a complication of treatment for congenital dislocation of the hip in young children: a clinical and experimental investigation. Can J Surg. 1969;12(1): 44-61. Epub 1969/01/01. PubMed PMID: 5762671

18. Clarke NM. Developmental dysplasia of the hip: diagnosis and management to 18 months. Instr Course Lect. 2014;63:307-11. Epub 2014/04/12. PubMed PMID: 24720316

19. Bulut O, Ozturk H, Tezeren G, Bulut S. Arthroscopic-assisted surgical treatment for developmental dislocation of the hip. Arthroscopy. 2005;21(5): 574-9. Epub 2005/05/14. doi: S0749806305000423 [pii]. doi: 10.1016/j.arthro. 2005.01.004. PubMed PMID: 15891724

20. Bolland BJ, Wahed A, Al-Hallao S, Culliford DJ, Clarke NM. Late reduction in congenital dislocation of the hip and the need for secondary surgery: radiologic predictors and confounding variables. J Pediatr Orthop. 2010; 30(7):676-82. Epub 2010/09/25. doi: 10.1097/BPO.0b013e3181 efb8c7. 01241398-201010000-00010 [pii]. PubMed PMID: 20864852

21. Tanaka T, Yoshihashi Y, Miura T. Changes in soft tissue interposition after reduction of developmental dislocation of the hip. J Pediatr Orthop. 1994; 14(1):16-23. Epub 1994/01/01. PubMed PMID: 8113365

22. Mason JB, McCarthy JC, O'Donnell J, Barsoum W, Mayor MB, Busconi BD, et al. Hip arthroscopy: surgical approach, positioning, and distraction. Clin Orthop Relat Res. 2003;406:29-37. Epub 2003/02/13. doi: 10.1097/01.blo. 0000043041.84315.cc. PubMed PMID: 12578997

23. Ozturk H, Oztemur Z, Bulut O, Tezeren G, Bulut S. Arthroscopic-assisted surgical treatment for developmental dislocation of the hip before the age of 18 months. Arch Orthop Trauma Surg. 2013;133(9):1289-94. Epub 2013/ 06/04. doi: 10.1007/s00402-013-1781-y. PubMed PMID: 23728833

24. Roy DR. The use of hip arthroscopy in the management of the pediatric hip. J Hip Preserv Surg. 2016;3(2):97-107. Epub 2016/09/02. doi: 10.1093/ jhps/hnv070. PubMed PMID: 27583144; PubMed Central PMCID: PMC5005042

\section{Submit your next manuscript to BioMed Central and we will help you at every step:}

- We accept pre-submission inquiries

- Our selector tool helps you to find the most relevant journal

- We provide round the clock customer support

- Convenient online submission

- Thorough peer review

- Inclusion in PubMed and all major indexing services

- Maximum visibility for your research

Submit your manuscript at www.biomedcentral.com/submit

) Biomed Central 